CARNETS DE Carnets de géographes

GÉOGRAPHES.

$1 \mid 2010$

Varia

\title{
Le pavillon et l'isoloir
}

Pourquoi une géographie sociale et électorale des espaces périurbains français?

Jean Rivière

\section{OpenEdition}

\section{Journals}

Édition électronique

URL : http://journals.openedition.org/cdg/2279

DOI : $10.4000 /$ cdg. 2279

ISSN : 2107-7266

Éditeur

UMR 245 - CESSMA

Référence électronique

Jean Rivière, "Le pavillon et l'isoloir », Carnets de géographes [En ligne], 1 | 2010, mis en ligne le 01 octobre 2010, consulté le 25 septembre 2020. URL : http://journals.openedition.org/cdg/2279 ; DOI : https://doi.org/10.4000/cdg.2279

Ce document a été généré automatiquement le 25 septembre 2020

\section{(c) (i) (3) $\Theta$}

La revue Carnets de géographes est mise à disposition selon les termes de la Licence Creative Commons Attribution - Pas d'Utilisation Commerciale - Pas de Modification 4.0 International. 


\section{Le pavillon et l'isoloir}

Pourquoi une géographie sociale et électorale des espaces périurbains français?

Jean Rivière

«On enregistre peu de différences entre le vote des banlieues ouvrières pavillonnaires ou non, et une enquête récente [...] a montré qu'au niveau des bureaux de vote, on n'en enregistre guère non plus entre quartiers pavillonnaires et grands ensembles » (Raymond, 1966).

"Même banlieue nord de Paris, même commune mais des choix politiques aux antipodes. [...] D'un côté, le quartier du Vert-Galant, une zone de pavillons assez modestes, où Nicolas Sarkozy et Jean-Marie Le Pen obtiennent au total $50 \%$ des suffrages, contre un peu plus de $23 \%$ pour Ségolène Royal. De l'autre côté, un quartier sensible, appelé Fontaine Mallet, où Ségolène Royal recueille $44 \%$ des suffrages, contre $27 \%$ au total pour MM. Le Pen et Sarkozy » (Bronner, 2007).

1 Quatre décennies se sont écoulées entre ces deux analyses. Que s'est-il passé entretemps? C'est à cette question que j'ai tenté d'apporter quelques éléments de réponse dans le cadre d'une thèse de géographie récemment soutenue à l'Université de Caen (Rivière, 2009). Pour cela, ce travail invite à une exploration des rapports entre le processus de périurbanisation, la redistribution des cartes de la division sociale de l'espace qu'il entraîne et les comportements électoraux que ces mutations participent à produire dans trois aires urbaines moyennes (Caen, Metz et Perpignan) exemplaires de la diversité des situations périurbaines françaises. Plus largement, c'est la question du changement social qui est au cœur de l'analyse, et ce dans des espaces généralement considérés comme l'univers des couches moyennes par excellence (Jaillet, 2004).

Cependant et à la différence des «aventuriers du quotidien » des années 1970-1980 et de leurs trajectoires ascendantes (Bidou, 1984), les couches moyennes contemporaines sont plutôt vues par les chercheurs en sciences sociales comme " déstabilisées ou à la dérive, repliées sur elles-mêmes ou sécessionnistes" (Bacqué \& Vermeersch, 2007). Depuis le scrutin présidentiel de 2002 et le référendum sur le Traité constitutionnel européen de 2005, nombre de travaux ont justement souligné certaines spécificités supposées des choix électoraux des habitants des espaces périurbains, qui tendraient à se distinguer des comportements adoptés par les citadins dans l'isoloir (Lévy, 2003). La notion de «gradient d'urbanité » y est invoquée pour expliquer ce clivage croissant qui 
traduirait l'idée que la localisation résidentielle dans tel ou tel type d'espace (central ou périurbain) résulte du système de valeurs des habitants (rapports au Monde et à l'Autre), ces valeurs s'exprimant aussi dans leurs bulletins de vote (Ripoll \& Rivière, 2007). Afin de démontrer que la seule localisation résidentielle périurbaine ne permet en rien d'éclairer les choix électoraux qui y ont lieu, cette thèse se propose d'analyser la production des votes en la ré-encastrant avec les appartenances et trajectoires socio-résidentielles des habitants d'une part, et en la saisissant là où elle fait sens d'autre part.

3 Pour éviter les pièges de l'approche surplombante, c'est la quête du réalisme sociologique qui a été privilégiée dans la manière de comprendre les choix électoraux des périurbains, d'où le recours à un « métissage méthodologique » (Passeron, 1997) qui repose sur l'idée de complémentarité des matériaux, des méthodes et des techniques. Après une première partie historiographique qui se propose d'étudier les regards scientifiques portés sur les votes des périurbains, la deuxième partie se penche sur les effets sociaux et électoraux de quatre décennies de périurbanisation. Le propos s'appuie pour cela sur l'analyse secondaire de données (recensements de l'INSEE de 1968 à 2006, résultats électoraux de 1969 à 2007) agrégées selon plusieurs échelles géographiques et traitées grâce aux outils de la statistique multivariée couplés à des représentations cartographiques. La troisième partie met ensuite les votes des périurbains à l'épreuve de leurs appartenances sociales et résidentielles via l'exploitation de questionnaires «sortie des urnes » collectés à l'occasion du scrutin présidentiel de 2007 dans huit communes emblématiques des trois aires urbaines étudiées. Enfin, la quatrième partie traite de la construction de la question de l'urbanisation comme enjeu politique local grâce à une enquête de terrain (entretiens, observations) conduite lors des municipales de 2008 dans deux communes de la périphérie caennaise qui constituent des idéaux-types des configurations dégagées jusqu'alors.

4 Contrairement aux sondages d'opinion nationaux qui extirpent les enquêtés de leurs sphères (locales) de socialisation tout autant qu'ils leur imposent de trancher sur des enjeux potentiellement extérieurs à leurs propres préoccupations, cette thèse tente ainsi de contribuer au renouveau des approches contextuelles des pratiques électorales, à l'image de travaux récemment menés dans un quartier populaire de grands ensembles (Braconnier \& Dormagen, 2007) ou dans une zone pavillonnaire urbaine de « petits-moyens » (Cartier et al., 2008). Pour éviter les postures normatives, il est en effet urgent de rappeler que des structures sociales pèsent sur les pratiques des acteurs, y compris derrière les rideaux de l'isoloir.

Fiche informative

Thèse disponible en ligne :

https://tel.archives-ouvertes.fr/file/index/docid/460099/filename/

Le_pavillon_et_l_isoloir.pdf

Discipline

Géographie

Directeurs

Robert Hérin, directeur (Caen Basse-Normandie) Michel Bussi, co-directeur (Rouen) 


\section{Université \\ Caen-Basse Normandie}

Membres du jury de thèse, soutenue le 30 novembre 2009

- Mme Martine Berger, Professeure de géographie à l'université de Paris I

Panthéon- Sorbonne, UMR LADYSS 7533 CNRS (Rapporteur)

- M. Martin Vanier, Professeur de géographie à l'université Joseph Fourrier de Grenoble I, UMR PACTE 5194 CNRS (Rapporteur)

- M. Patrick Lehingue, Professeur de science politique à l'université de Picardie Jules Verne, UMR CURAPP 6054 CNRS (Examinateur)

- M. Michel Bussi, Professeur de géographie à l'université de Rouen, UMR IDEES 6266 CNRS (Co-directeur de thèse)

- M. Robert Hérin, Professeur émérite de géographie à l'université de Caen BasseNormandie, UMR ESO 6590 CNRS (Directeur de thèse)

Situation professionnelle actuelle

Chercheur Post-Doctoral à l'Université de Rouen, membre associé à l'UMR ESO 6590 CNRS

Contact

jean.riviere[at]unicaen.fr

\section{BIBLIOGRAPHIE}

BACQUÉ M.-H., VERMEERSCH S., 2007, Changer la vie ? Les classes moyennes et l'héritage de mai 68, Paris : Éditions de l'atelier.

BIDOU C., 1984, Les Aventuriers du quotidien. Essai sur les nouvelles classes moyennes, Paris :

P.U.F.

BRACONNIER C., DORMAGEN J.-Y., 2007, La démocratie de l'abstention, Paris : Gallimard. BRONNER L., 2007, « Pavillons contre HLM, le clivage des banlieues », in Le Monde, 24 avril.

CARTIER M., COUTANT I., MASCLET O., SIBLOT Y., 2008, La France des « petits-moyens ». Enquête sur la banlieue pavillonnaire, Paris : La Découverte. CHAUVEL L., 2006, Les classes moyennes à la dérive, Paris : Le Seuil.

JAILLET M.-C., 2004, « L'espace périurbain : un univers pour les classes moyennes », in Esprit, 303, 43-65.

LÉVY J., 2003, « Périurbain : le choix n'est pas neutre », in Pouvoir local, 56(1), 35-42.

PASSERON J.-C., 1997, « Le modèle, l'enquête et le récit », in MAYER N., Les modèles explicatifs du vote, Paris : Presses de Sciences Po, 19-43.

RAYMOND M.-G., 1966, La politique pavillonnaire, Paris : CRU. 
RIPOLL F., RIVIÈRE J., 2007, « La ville dense comme seul espace légitime ? Analyse critique d'un discours dominant sur le vote et l'urbain », in Les annales de la recherche urbaine, 102, 121-130.

RIVIÈRE J., 2009, Le pavillon et l'isoloir. Géographie sociale et électorale des espaces périurbains français (1968-2008). À travers les cas de trois aires urbaines moyennes (Caen, Metz, Perpignan), Thèse de doctorat en géographie sous la direction de R. HÉRIN et de M. BUSSI, Université de Caen Basse-Normandie, 2 tomes.

INDEX

Thèmes: Carnets de soutenances 